\title{
Low-Offset In-Plane Sensitive Hall Arrangement ${ }^{\dagger}$
}

\author{
Siya Lozanova, Ivan Kolev, Avgust Ivanov and Chavdar Roumenin * \\ Institute of Robotics at Bulgarian Academy of Sciences, Sofia, Bulgaria; lozanovasi@abv.bg (S.L.); \\ ivankolew91@gmail.com (I.K.); avgust.ivanov@abv.bg (A.I.) \\ * Correspondence: roumenin@bas.bg; Tel.: +359-2-870-3361 \\ † Presented at the Eurosensors 2018 Conference, Graz, Austria, 9-12 September 2018. \\ Published: 21 December 2018
}

\begin{abstract}
A novel in-plane sensitive Hall arrangement consisting of two identical $n$-Si three-contact (3C) elements and realized in a common technological process, is presented. In the solution, the minimization of the offset and its temperature drift is achieved by cross-coupling of the outer device contacts. This terminals' connection provides equalizing currents between the two substrates which strongly compensate the inevitable difference in the electrical conditions in the two parts of the arrangement. As a result, the residual offset of both integrated Hall elements at the output $V_{\text {out }}(0)$ and its temperature drift are strongly minimized. The residual offset is about 160 times smaller than the single-configuration one. The obtained output voltage-to-residual offset ratio at sensitivity of $\mathrm{S}_{\mathrm{RI}} \approx 98 \mathrm{~V} / \mathrm{AT}$ is very promising, reaching $6 \times 10^{3}$ at temperature $T=40^{\circ} \mathrm{C}$ and induction $1 \mathrm{~T}$. As a result, increased metrological accuracy for numerous applications is achieved. For a first time through the novel arrangement a suppression of sensitivity in the presence of external magnetic field could be achieved in order to obtain permanent offset information. This is one of the key results in the Hall deviceinvestigation.
\end{abstract}

Keywords: in-plane sensitive Hall arrangement; offset minimization; silicon three-contact Hall element

\section{Introduction}

Offset is an enormous disadvantage of Hall effect devices with differential output measured in the absence of a magnetic-field only. This drawback changes the sensor's output in an unpredictable way. The most probable reasons for the occurrence of the offset are: the electrical asymmetry (resulting from geometrical errors in the masks' positioning in the technological production process) caused by the geometry in the location of the peripheral contacts with respect to the central one, the inevitable structure damages and imperfections of the silicon substrates, the mechanical and temperature stress and strain (caused most often by the chip's capsulation) and more, [1-5]. This problem is crucial in many areas of sensor applications, like robotics, mechatronics, navigation, contactless automation, position sensors, angular displacement detection, automobile industry including ABS systems. For example, the 3C, 4C, 5C and 6C in-plane sensitive Hall devices have relatively high offset, larger than the offset of the orthogonal Hall plates, alongside with substantial temperature drift. These defects seriously impede measurement accuracy. The most common solutions removing these problems are very complicated and costly-for example, dynamic offset cancelation by various current spinning techniques. Such approach does not match the ordinary Hall element and its transduction principle. This paper suggests a novel simple, but sufficiently effective in-plane sensitive silicon Hall arrangement, ensuring low offset and low temperature drift. 


\section{Sensor Layout and Operation Principle}

The novel Hall configuration consists of two identical $n$-Si substrates, parallel to one another, Figure 1. On the top surface of each of the structures, three identical rectangular $n^{+}-n$ contacts are formed $-C_{1}, C_{2}, C_{3}$ and $C_{4}, C_{5}, C_{6}$, respectively, Figures 1 and 2 . The supply source Es is connected to both middle contacts $\mathrm{C}_{2}$ and $\mathrm{C}_{5}$.

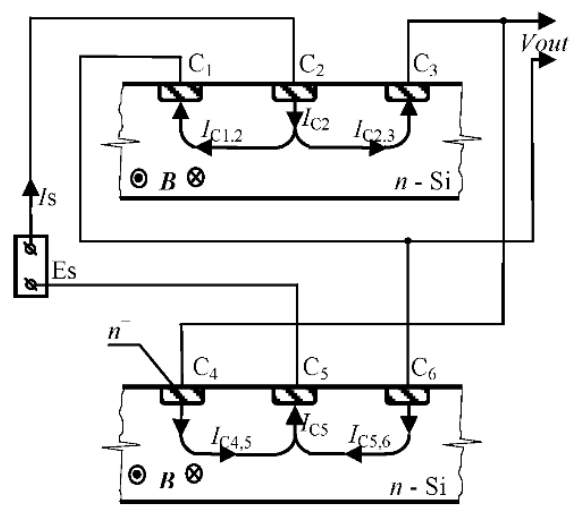

Figure 1. The novel Hall arrangement.

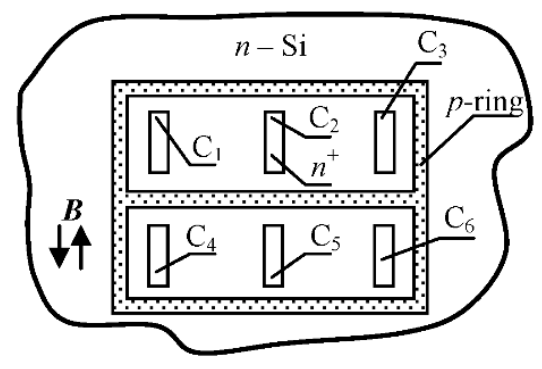

Figure 2. Top-view of the Hallarchitecture.

The differential output $V_{\text {out }}(B)$ of the Hall arrangement is between contacts $C_{1}$ and $C_{3}$ (or $C_{4}$ and $C_{6}$ ). The measured magnetic field $B$ is parallel to the long sides of the contacts. When power supply Es is fed, by cross-coupling of the outer contacts $C_{1}-C_{6}$ and $C_{3}-C_{4}$, in the substrate bulks, two equal and opposite currents $I_{\mathrm{C}_{2}}$ and $-I_{\mathrm{c} 5}$ start to flow, $I_{\mathrm{c} 2}=\left|-I_{\mathrm{c} 5}\right| \equiv I_{\mathrm{s}}$, Figure 1 .

The experimental prototype has been fabricated using part of the processing steps applied in bipolar IC technology. The resistivity of the $n$-Si substrate is $\varrho \approx 7.5 \Omega . \mathrm{cm}\left(n 0 \approx 4 \times 10^{15} \mathrm{~cm}^{-3}\right)$. Similarly to [6], four masks are used. The size of contacts $C_{1} \ldots C_{6}$ is $50 \times 10 \mu \mathrm{m}^{2}$, the distance between the central and the outer contacts is $25 \mu \mathrm{m}$ and the width of the $p$-ring on the chip surface is about 30 $\mu \mathrm{m}$. The penetration of the current trajectories into the $n$-Si substrates reaches a depth of $30-40 \mu \mathrm{m}$. The operational volume of the device is about $100 \times 70 \times 40 \mu \mathrm{m}^{3}[7]$.

The action of the in-plane Hall arrangement is the following. When the two central contacts $C_{2}$ and $\mathrm{C}_{5}$ are connected to supply ES, in the bulk of the two substrates, two opposite-direction and equal-value current components, $I_{c 2,1}$ and $-I_{C 2,3}, I_{c 5,4}$ and $-I_{c 5,6,}$ respectively, flow. The planar central contacts $C_{2}$ and $C_{5}$, as well as the outer contacts $C_{1}$ and $C_{3}$, and $C_{4}$ and $C_{6}$ respectively, form equipotential planes where, in the absence of magnetic field, $B=0$, the currents flowing through them are always perpendicular to the upper side of the chip. The current lines penetrate deep into their volumes. The depth at fixed concentration of the dopant donors ND in the substrates depends on the ratio between the width of the central contacts and the distance between them and the outer electrodes. The maximal value of the depth in $n$-Si at $\mathrm{ND}_{D} \approx 10^{15} \mathrm{~cm}^{-3}$ constitutes about $40 \mu \mathrm{m}$. Therefore, the trajectories of the carriers are curvilinear, [1-3]. In the general case, at the output $V_{\text {out }}(0)$, in the absence of magnetic field, $B=0$, parasitic output offset $V_{\text {out }}(0) \neq 0$ is available, which is not related with metrology. In the proposed solution, Figure 1, the minimization and/or removal of 
the offset is achieved by the direct connection of contacts $C_{1}$ and $C_{6}$, and $C_{3}$ and $C_{4}$,respectively. This short-circuit results in the flowing of compensation equalizing currents between the two identical Hall structures in the absence of magnetic field, $B=0$.

The application of the measured magnetic field $B \neq 0$ in parallel to the long sides of the contacts results in lateral deflection of the current lines along the entire length of their non-linear trajectory. This is due to the action of Lorentz forces $F_{\mathrm{L}, \mathrm{i}}, \boldsymbol{F}_{\mathrm{L}}=q \boldsymbol{V}_{\mathrm{dr}} \times \boldsymbol{B}$, where $q$ is the electron's elementary charge, and $V_{\mathrm{dr}}$ is the vector of the mean drift velocity of the electrons in the substrates, [1,3]. Of key importance is the effect of the force $F_{\mathrm{L}}$ on the components $I_{2}$ and $I_{5}$ through the central contacts $\mathrm{C}_{2}$ and $\mathrm{C}_{5}$. As a result of the Lorentz deflection, the non-linear trajectories shrink and expand, respectively. For this reason, on planar contacts $C_{1}$ and $C_{3}$, and $C_{4}$ and $C_{6}$, Hall potentials $V_{\mathrm{H}, \mathrm{C}}(\boldsymbol{B})=$ $V_{\mathrm{H}, \mathrm{C} 6}(\boldsymbol{B})$ and $-V_{\mathrm{H}, \mathrm{C4}}(\boldsymbol{B})=-V_{\mathrm{H}, \mathrm{C} 3}(\boldsymbol{B})$ are generated simultaneously. Actually, the measured magnetic field $\boldsymbol{B}$ disturbs the electric symmetry of the current trajectories with respect to the central contacts. Therefore, on the differential output $V_{\mathrm{out}}(\boldsymbol{B})$ of the arrangement, Figure 1, Hall voltage $V_{\mathrm{H}}(\boldsymbol{B}) \equiv V_{\mathrm{H}, \mathrm{Cl}-}$ ${ }_{\mathrm{C}}(B)$ arises. This signal is a linear and odd function of the strength of the supply current and the magnetic field $B$. On the respective outer contacts $C_{1}-C_{6}$ and $C_{3}-C_{4}$, Hall potentials with the same sign and value are generated. The solution from Figure 1 reduces drastically the offset. The configuration features yet another advantage, which is one of the key results in this investigation. If contacts $C_{1}$ and $C_{4}$, and $C_{3}$ and $C_{6}$ are connected using electronic switch, for example, then Hall potentials (Hall voltage) compensation (nulling) occurs:

$$
V_{\mathrm{H}, \mathrm{C} 1}(\boldsymbol{B})=|-V \mathrm{H}, \mathrm{C} 4(\boldsymbol{B})| \text { and } V_{\mathrm{H}, \mathrm{C} 3}(\boldsymbol{B})=\left|-V_{\mathrm{H}, \mathrm{C} 6}(\boldsymbol{B})\right| .
$$

As a result, the magnetosensitivity in the two identical configurations is lacking. The only remaining signal is the parasitic offset. In this case the output is between contacts $C_{1}$ and $C_{3}$ or $C_{4}$ and $\mathrm{C}_{6}$. The purpose is to achieve at any time information about the negative offset in output signal, regardless the available external magnetic field. Such approach is proposed for the first time in the sensorics of Hallelements.

\section{Results}

The output characteristics $V_{\text {out }}(\boldsymbol{B})$ of the microdevice are presented in Figure 3. The obtained relative current magnetosensitivity is $\mathrm{S}_{\mathrm{RI}} \approx 98 \mathrm{~V} / \mathrm{AT}$. The non-linearity $\mathrm{NL}$ does not exceed $0.6 \%$ within the range $0.5 \mathrm{~T} \div-0.5 \mathrm{~T}$.

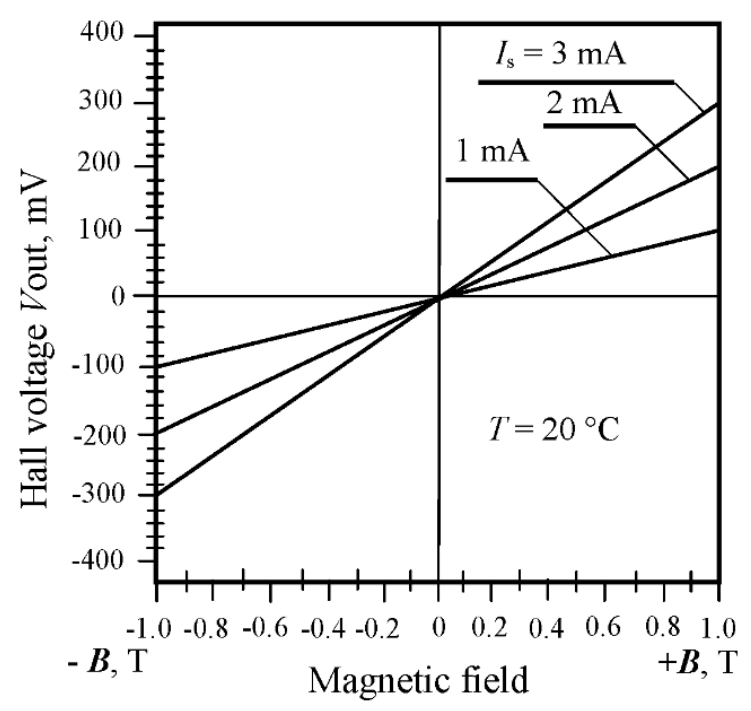

Figure 3. Output characteristics $V_{\text {out }}(\boldsymbol{B})$.

The temperature dependences of the single-substrate offset $V_{\mathrm{ss}}(0)$ and the residual offset $V_{\text {out }}(0)$ of the arrangement, at a supply current $I_{\mathrm{s}}=3 \mathrm{~mA}$ and field $B=0$ in the temperature range $-10 \leq T \leq$ 
$80^{\circ} \mathrm{C}$, are shown in Figure 4. The temperature coefficient of the offset drift reaches no more than $0.1 \% /{ }^{\circ} \mathrm{C}$. The obtained output voltage-to-residual offset ratio, for example at $T=40{ }^{\circ} \mathrm{C}$, reaches around $6 \times 10^{3}$ at induction $B=1 \mathrm{~T}$. This is a very promising result. At first approximation the residual offsets measured at field $B=0$ and induction $B=0.35 \mathrm{~T}$ are on a straight line, Figure 5 .

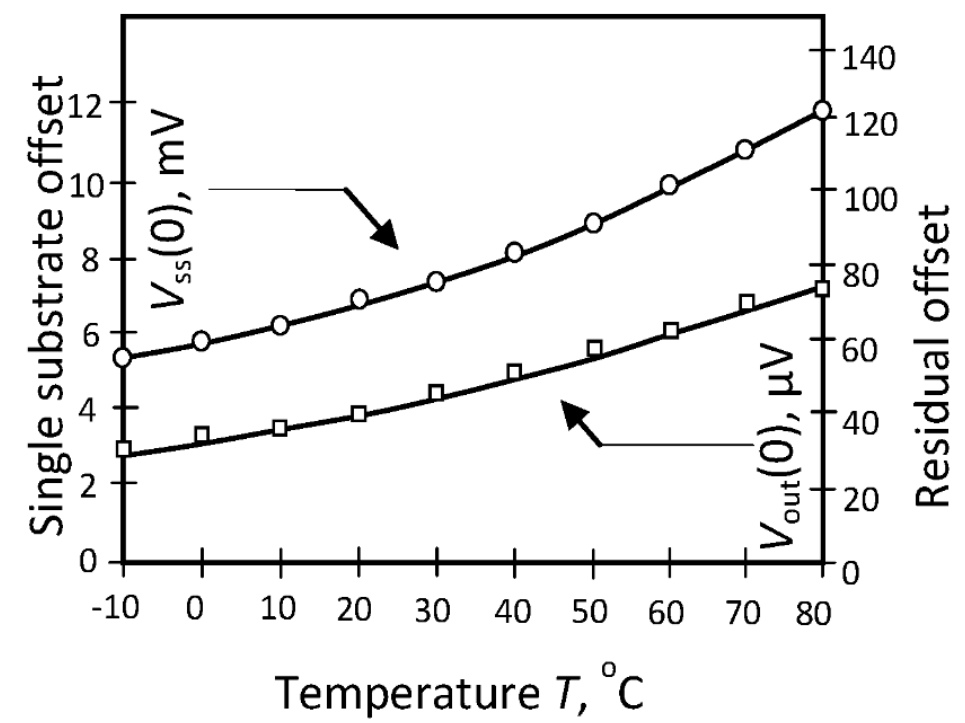

Figure 4. Temperature dependences of single offset $V_{\mathrm{ss}}(0)$ and residual offset $V_{\mathrm{out}}(0)$ at a supply $I_{\mathrm{s}}=3 \mathrm{~mA}$.

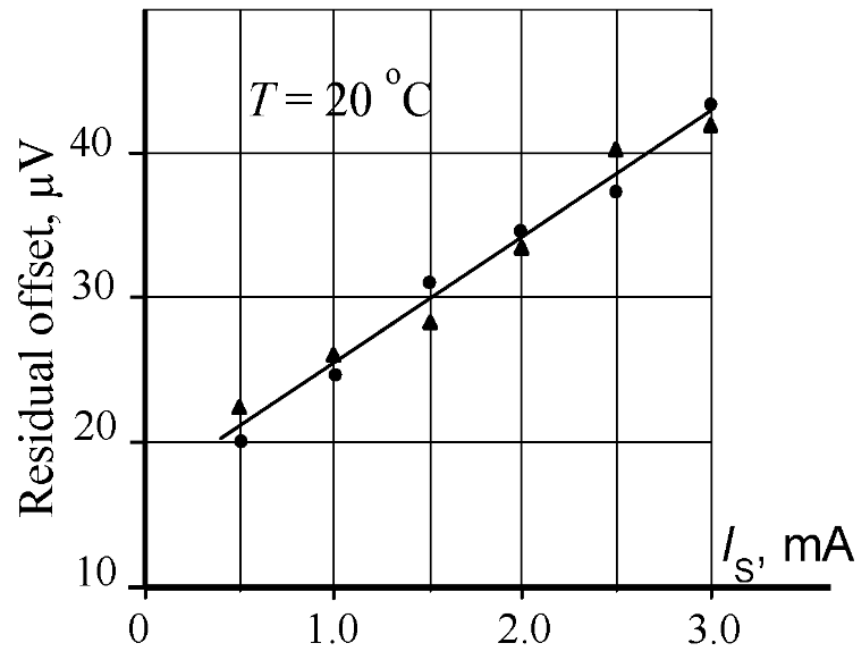

Figure 5. Residual offsets at field $B=0(\bullet)$ and induction $B=0.35 \mathrm{~T}(\mathbf{\Delta})$.

\section{Conclusions}

The new solution is a combination of innovative circuit technology and device design through which, with one supply current, high magnetosensitivity in both substrates is generated. The original connection of the outer contacts reduces substantially the inevitable parasitic offset by flowing of equalizing currents.

For the first time in the Hall effect sensors, through the new arrangement, complete suppression of magnetosensitivity in the presence of magnetic field could be achieved. This result follows from the identity of the two $3 \mathrm{C}$ structures and the common supply current. This allows to be obtained at any time information about the value and sign of the offset for the purpose of its extraction from the sensor's output signal. The proposed in-plane sensitive Hall arrangement features multi-purpose industrial applicability. 
Acknowledgments. This work was supported by Sci. Res. Fund under project No. DN 07/18-15.12.2016.

\section{References}

1. Roumenin, C. Solid State Magnetic Sensors; Elsevier: Amsterdam, The Netherlands, 1994.

2. Kaufmann, T. On the Offset and Sensitivity of CMOS-Based Five-Contact Vertical Hall Devices; MEMS Technology and Engineering; Der Andere Verlag: Uelvesbull, Germany, 2013; Volume 21, p. 147.

3. Lozanova, S.V.; Roumenin, C.S. Parallel-field silicon Hall effect microsensors with minimal design complexity. IEEE Sens. J. 2009, 9, 761-766.

4. Schurig, E.; Schott, C.; Besse, P.-A.; Popovic, R.S. CMOS integrated vertical Hall sensor with low offset. In Proceedings of the 16th European Conference on Solid-State Transducers, Prague, Czech Republic, 15-18 September 2002; pp. 868-871.

5. Schurig, E.; Schott, C.; Besse, P.-A.; Demierre, M.; Popovic, R.S. $0.2 \mathrm{mT}$ residual offset of CMOS integrated vertical Hall sensors. Sens. Actuators A 2004, 110, 98-104.

6. Schott, C.; Popovic, R. Integrated 3-D Hall magnetic field sensor. In Proceedings of the Transducers'99, Sendai, Japan, 7-10 June 1999; Volume 1, pp.168-171.

7. Lozanova, S.; Noykov, S.; Ivanov, A.; Roumenin, C. In-plane magnetosensitive double Hall device. In Proceedings of the 2017 XXVI International Scientific Conference Electronics (ET), Sozopol, Bulgaria, 1315 September 2017; pp. 19-21.

(C) 2018 by the authors. Licensee MDPI, Basel, Switzerland. This article is an open access article distributed under the terms and conditions of the Creative Commons Attribution (CC BY) license (http://creativecommons.org/licenses/by/4.0/). 